\title{
Aging and visual 3-D shape recognition from motion
}

\author{
J. Farley Norman ${ }^{1}$ • Olivia C. Adkins ${ }^{2}$ - Catherine J. Dowell ${ }^{1}$ Stevie C. Hoyng ${ }^{1}$ • \\ Lindsey M. Shain ${ }^{1} \cdot$ Lauren E. Pedersen $^{1} \cdot$ Jonathan D. Kinnard $^{3}$ • \\ Alexia J. Higginbotham ${ }^{1}$ - Ashley N. Gilliam ${ }^{1}$
}

Published online: 25 July 2017

(C) The Psychonomic Society, Inc. 2017

\begin{abstract}
Two experiments were conducted to evaluate the ability of younger and older adults to recognize 3-D object shape from patterns of optical motion. In Experiment 1, participants were required to identify dotted surfaces that rotated in depth (i.e., surface structure portrayed using the kinetic depth effect). The task difficulty was manipulated by limiting the surface point lifetimes within the stimulus apparent motion sequences. In Experiment 2, the participants identified solid, naturally shaped objects (replicas of bell peppers, Capsicum annuиm) that were defined by occlusion boundary contours, patterns of specular highlights, or combined optical patterns containing both boundary contours and specular highlights. Significant and adverse effects of increased age were found in both experiments. Despite the fact that previous research has found that increases in age do not reduce solid shape discrimination, our current results indicated that the same conclusion does not hold for shape identification. We demonstrated that aging results in a reduction in the ability to visually recognize 3-D shape independent of how the 3-D structure is defined (motions of isolated points, deformations of smooth optical fields containing specular highlights, etc.).
\end{abstract}

J. Farley Norman

Farley.Norman@wku.edu

1 Department of Psychological Sciences, Ogden College of Science and Engineering, Western Kentucky University, Bowling Green, KY 42101-2030, USA

2 Department of Psychological and Brain Sciences, Indiana University, Bloomington, IN 47405, USA

3 Carol Martin Gatton Academy of Mathematics and Science, Bowling Green, KY 42101-1031, USA
Keywords 3D perception: Depth and shape from X · aging · Motion: In Depth

From previous research (Norman et al., 2006, Experiment 1), we know that older adults can effectively discriminate 3-D shape (same vs. different judgments) from moving visual stimuli, at least under full cue conditions (solid objects, complete rotation in depth, shading, specular highlights, boundary contour deformations, etc.). In that experiment, there was no age effect at all; the older adults performed as well as the younger adults. In a later study, however (Norman et al., 2013), a substantial age-related deficit occurred when participants were required to recognize dotted surfaces where the only information available to support the participants' judgments was the movement of isolated points (Andersen, 1996; Braunstein, 1966; Norman \& Lappin, 1992; Todd \& Norman, 1991).

Previous research has found that older adults 1) can perform equivalently to younger adults when making relatively simple shape discriminations (same vs. different shape judgment), and 2) possess substantive deficits when recognizing objects whose shapes are portrayed by the conventional kinetic depth effect. At the moment, it is unclear what factor(s) account for the varying performances of older adults (sometimes exhibit as high a performance as younger adults, sometimes perform considerably worse than younger adults). One possibility for the varying outcomes was the presence of natural object boundary contours and specular highlights in the stimuli of Norman et al. (2006); these were not present in the motion displays used by Norman et al. (2013). Another possibility for the difference in outcome is the task itself, shape discrimination (Norman et al., 2006) versus shape recognition/identification (Norman et al., 2013). The goal of the current study was to resolve this ambiguity. The purpose of 
Experiment 1 was to replicate the findings of Norman et al. (2013) with a new sample of older adults. The purpose of Experiment 2 was to evaluate these older adults' abilities to recognize solid object shape from moving and nonmoving visual patterns containing all combinations of specular highlights and boundary contours (boundary contours only, specular highlights only, boundary contours combined with specular highlights). If the effect of aging obtained by Norman et al. (2013) was due to task demands associated with shape recognition, the current older adults' performance should be significantly worse than that of the younger adults in both of the current experiments. If the good performance exhibited by the older adults of Norman et al. (2006) was enabled by the presence of extra optical information (the specular highlights and boundary contours that occur for naturally shaped solid objects), then an adverse effect of age should occur in the current Experiment 1 but not in the current Experiment 2. The specific purpose of the current study was to evaluate these possibilities and determine older adults' true capabilities regarding shape identification/recognition.

\section{Experiment 1}

\section{Method}

Apparatus The visual stimuli were generated by an Apple PowerMacintosh G4 computer and displayed using a 22inch Mitsubishi Diamond Plus 200 color monitor $(1280 \times$ 1024 pixels).

Experimental stimuli The experimental stimuli were created in an identical manner to that used by Norman et al. (2013). On any given trial, one of three possible curved surfaces was presented for approximately 3 seconds ( 88 frames in the apparent motion sequences $\times 33.3 \mathrm{msec}$ per frame). The surfaces resembled "bullseyes," "snowflakes," and "egg crates" and were formed by modulating the surfaces in depth sinusoidally in radial or tangential directions (if $r$ and $\theta$ are polar coordinates, $\mathrm{z}=\sin [\mathrm{r}]$ or $\mathrm{z}=\sin [\theta]$ ), or as a function of Cartesian $\mathrm{x}$ and $y$ coordinates (e.g., $z=\sin [x]^{*} \sin [y]$ ). The surface stimuli possessed an average spatial frequency of 0.3 cycles/deg visual angle; the phases of the modulating sine waves were randomly determined on every trial so that each trial's stimulus was unique. The front-to-back depth of the corrugated surfaces was $1.0 \mathrm{~cm}$. The 3-D structure of each surface stimulus was defined by the projected (perspective) motions of 800 bright points (white points against a black background); the surfaces rotated/oscillated in depth \pm 22 degrees about a Cartesian vertical axis (total range of rotation was $44 \mathrm{deg}$ ). The surfaces rotated 2 degrees in depth at every frame transition.
Procedure As was the case in the study by Norman et al. (2013), we manipulated the difficulty of the recognition task by varying the surface point lifetime; each of the 800 points defining the 3-D surfaces would "survive" and move with an appropriate velocity across a limited number of frames $(4,8$, or 15 frames). Once a particular surface point reached its maximal lifetime, it would "die" and be moved to a randomlydetermined location in the succeeding frame. Limiting the point lifetime is an effective way of introducing "correspondence noise" into the stimuli: the lower the surface point lifetime, the more difficult the identification task (Norman, Dawson, \& Butler, 2000; Todd, 1985; van Damme \& van de Grind, 1996).

Fifteen trials were conducted for each participant for each of the 9 combinations of surface shape (snowflake, bullseye, eggcrate) and surface point lifetime (4, 8, or 15 frames); thus each participant made a total of 135 judgments. On each trial, the participant was required to identify the particular surface shape that was presented.

Participants There were a total of 28 participants: 14 older adults (mean age was 73.5 years, standard deviation $[\mathrm{SD}]=5.2$, range $67-81$ years) and 14 younger adults (mean age was 20.6 years, $\mathrm{SD}=2.3$, range $18-24$ years). The participants' visual acuity was good (mean acuity for the younger and older adults was -0.129 and -0.007 $\operatorname{logMAR}$, respectively). All participants gave written consent prior to participation in the experiment. The experiment was approved by the Western Kentucky University Institutional Review Board. Our research was performed in accordance with the Code of Ethics of the World Medical Association (Declaration of Helsinki).

\section{Results and discussion}

The participants' results are shown in Fig. 1; the younger and older participants' shape recognition accuracies are plotted as a function of surface point lifetime. As can be readily seen, the younger participants' shape recognition performance was substantially higher (31.5\% higher) than that exhibited by the older adults. A 2-way ANOVA (1 between-subjects factor, age, and 1 within-subjects factor, surface point lifetime) conducted upon the results shown in Fig. 1 revealed that there was a significant effect of age $\left(\mathrm{F}(1,26)=18.4, p<0.001 ; \eta^{2}=0.42\right)$. There was also a significant effect of surface point lifetime $(\mathrm{F}(2,52)=$ 80.9, $\left.p<0.000001 ; \eta_{\mathrm{p}}^{2}=0.76\right)$; the age $\mathrm{x}$ surface point lifetime interaction was not significant $(\mathrm{F}(2,52)<1.0, p=$ $\left.0.38 ; \eta_{\mathrm{p}}^{2}=0.036\right)$. The lack of an interaction demonstrates that the reductions in surface point lifetime had a similarly detrimental effect upon the shape recognition performance of both younger and older adults. 


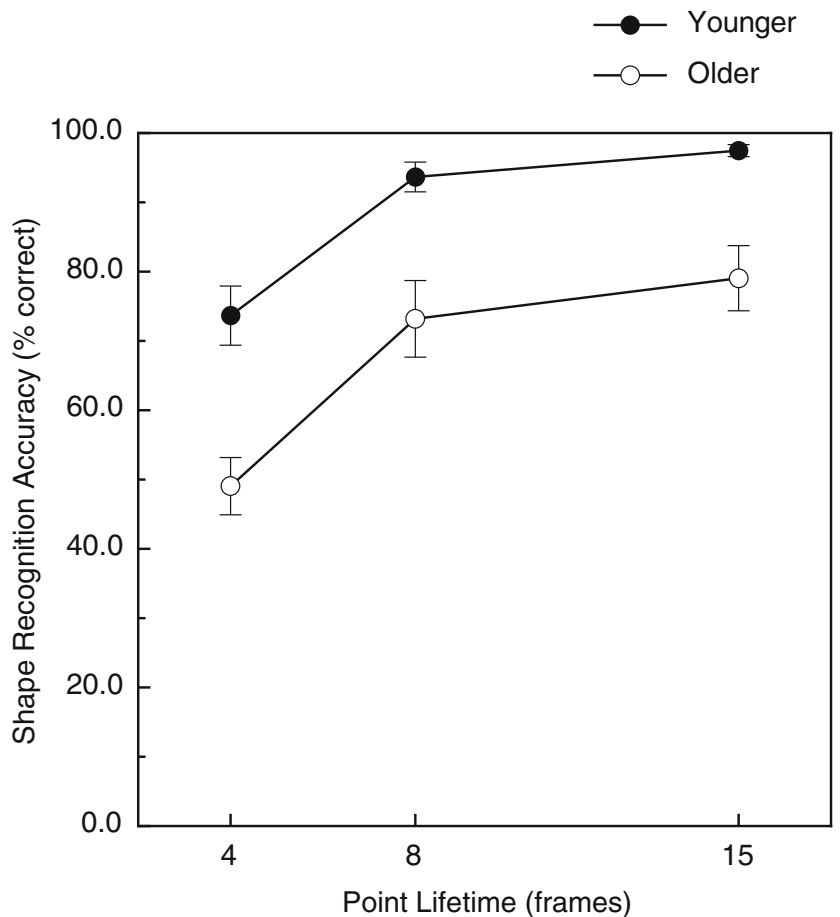

Fig. 1 Overall results for the younger and older adults in Experiment 1. The participants' shape recognition accuracies are plotted as a function of the surface point lifetime. The error bars indicate $\pm 1 \mathrm{SE}$.

\section{Experiment 2}

The results of Experiment 1 (Fig. 1) demonstrate that our current sample of 14 older adults, as a whole, perform more poorly than younger adults when recognizing the shape of surfaces whose 3-D structure is defined solely by the motion of projected points. The purpose of Experiment 2 was to determine whether this same group of older adults also would exhibit reduced shape recognition for more ecologically valid solid objects whose surface structure was defined by occlusion boundary contours and/or specular highlights. When solid (and shiny) objects rotate in depth, their boundary contours and specular reflections deform over time (Fig. 2). Boundary contours and specular highlights (and their temporal deformations) are effective sources of optical information to support the perception of 3-D shape (Fleming, Torralba, \& Adelson, 2004; Kennedy, 1974; Kennedy \& Silver, 1974; Koenderink \& van Doorn, 1976; Koenderink, 1984; Koenderink, Kappers, Todd, Norman, \& Phillips, 1996; Norman \& Raines, 2002; Norman, Todd, \& Phillips, 1995; Norman, Todd, \& Orban, 2004; Norman \& Wiesemann, 2007). Indeed, Norman, Todd, and Orban (2004) have demonstrated that stimulus displays containing specular highlights (and their deformations) produce the highest (i.e., best) shape discrimination performance. In a previous study (Norman et al., 2006), utilizing full-cue conditions (solid shiny objects that rotated in depth producing temporal deformations of occlusion boundary contours and specular highlights), older adults performed as well as

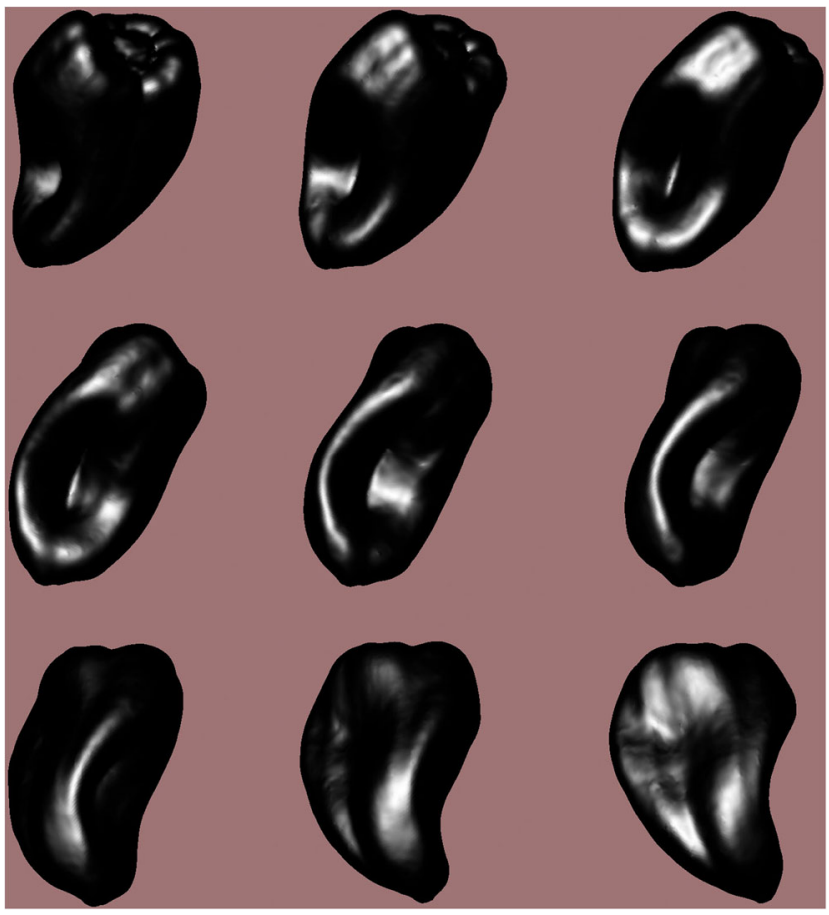

Fig. 2 The deformations of occlusion boundary contours and specular highlights that accompany the rotation of a solid object in depth. In this figure, a naturally shaped object (bell pepper 14, 1 of the 12 stimulus objects utilized in the current experiment) rotates in depth about a Cartesian vertical axis (in a counterclockwise direction, if considering the object from above); the sequence of frames from an apparent motion sequence proceeds from the first frame depicted (upper left) to the last frame depicted (bottom right). Between each of the 9 frames/ views that are illustrated, the object rotated 20 degrees in depth (in the actual experiment, objects rotated 1.5 degrees at each frame transition). Note that over time, both the occlusion boundary contour and the pattern of specular highlights deform in a complex manner, e.g., the pattern(s) of specular highlights merge, break apart, change shape, disappear, etc.

younger adults in a shape discrimination task (same vs. different shape discrimination). A primary goal of the current experiment was to determine whether the occlusion boundary contours and specular highlights associated with solid objects would similarly enable older adults to perform shape recognition/identification as well as younger adults.

\section{Method}

Apparatus The computer-rendered visual stimuli were generated by an Apple Mac Pro computer (Dual Quad-Core processors, with ATI Radeon HD 5770 hardware-accelerated graphics) and displayed on the same monitor used in Experiment 1 (Mitsubishi Diamond Plus 200). The monitor was located at a $100-\mathrm{cm}$ viewing distance.

Experimental stimuli The objects used in this experiment were solid naturally shaped replicas of bell peppers 
(Capsicum annuum) that have been utilized in previous experiments (Norman, Cheeseman, Adkins, et al. 2015; Norman, Adkins, Dowell, Hoyng, et al., 2017). These objects (13-24, different from the original set of 12 bell peppers used by Norman, Norman, Clayton, Lianekhammy, \& Zielke, 2004) are shown in Fig. 3. To create the stimulus displays used in the current experiment, the bell pepper replicas were first scanned using a laser scanner (NextEngine model 2020i Desktop 3D scanner). The resolution of the resulting scanned object copies was very good; the object surfaces were defined, on average, by approximately 147,900 triangular faces. There were three types of experimental stimuli (Fig. 4): objects defined by 1) occlusion boundary contours and specular highlights (condition BCSH), 2) occlusion boundary contours only (condition $\mathrm{BC}$ ), and 3) specular highlights only (condition $\mathrm{SH}$ ). The experimental stimuli were rendered with OpenGL (Shreiner, 2010) using a traditional reflectance model (Hughes et al., 2014) where stimulus image intensities are determined by ambient, diffuse, and specular coefficients (in the current study, the ambient and diffuse coefficients were all set to zero, because the 3-D structures of the experimental stimuli were defined solely by boundary contours and specular highlights). The parameters used for the rendering of the black shiny surfaces were the same as those used in the analogous conditions of Norman, Phillips, et al. (2016): a shininess exponent of 20.0 and a simulated white point light source located up and to the left of the stimulus objects (slant of light source was 30 degrees). As shown in the lower left panel of Fig. 4, stimulus objects in the boundary-only condition (BC) were depicted as solid black silhouettes.

Procedure There were a total of six experimental conditions formed by the orthogonal combination of three stimulus types (BCSH, BC, \& $\mathrm{SH}$ ) and the presence or

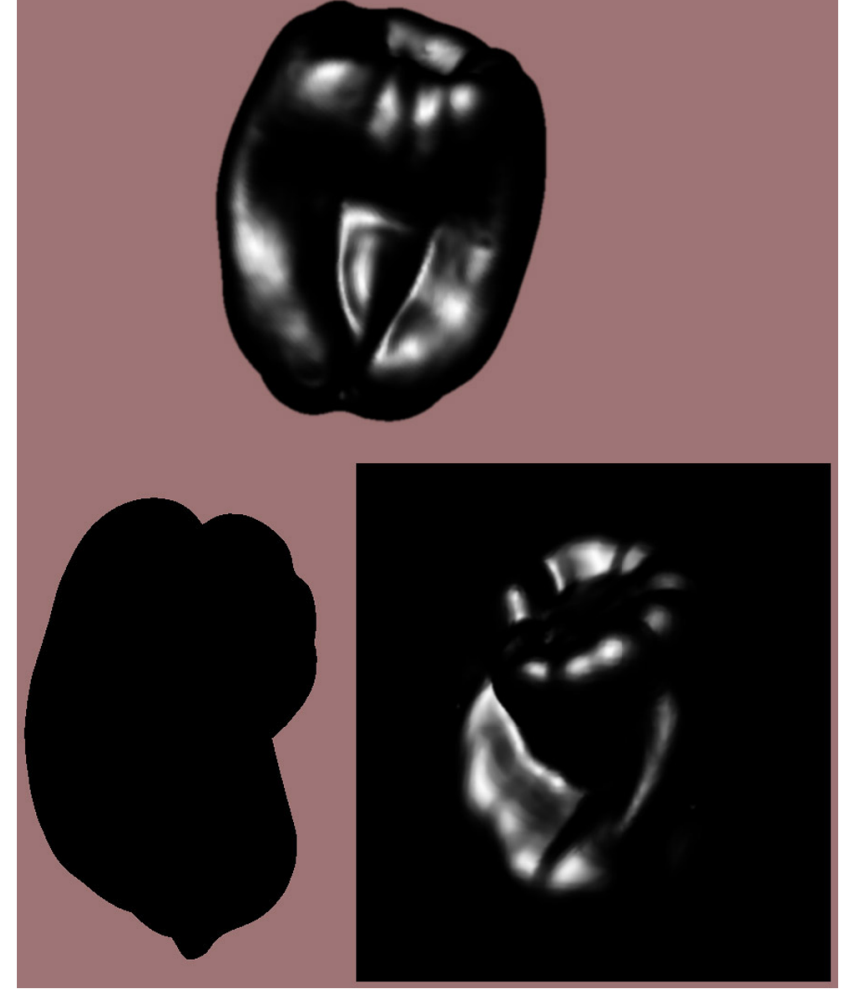

Fig. 4 Example stimuli illustrating the three stimulus types (i.e., optical information types). At the top, an object (bell pepper 22) is depicted with both occlusion boundary contours and specular highlights (condition BCSH). At the bottom-left, an object (bell pepper 16) is depicted only by occlusion boundary contours (condition $\mathrm{BC}$ ). At the bottom-right, an object (bell pepper 13) is depicted only by specular highlights (condition $\mathrm{SH})$.

absence of object motion. In the moving object conditions, the objects rotated in depth about a Cartesian vertical axis (at each frame transition, the objects rotated by $1.5 \mathrm{deg}$; the frame update rate was $75 \mathrm{~Hz}$ ). In all

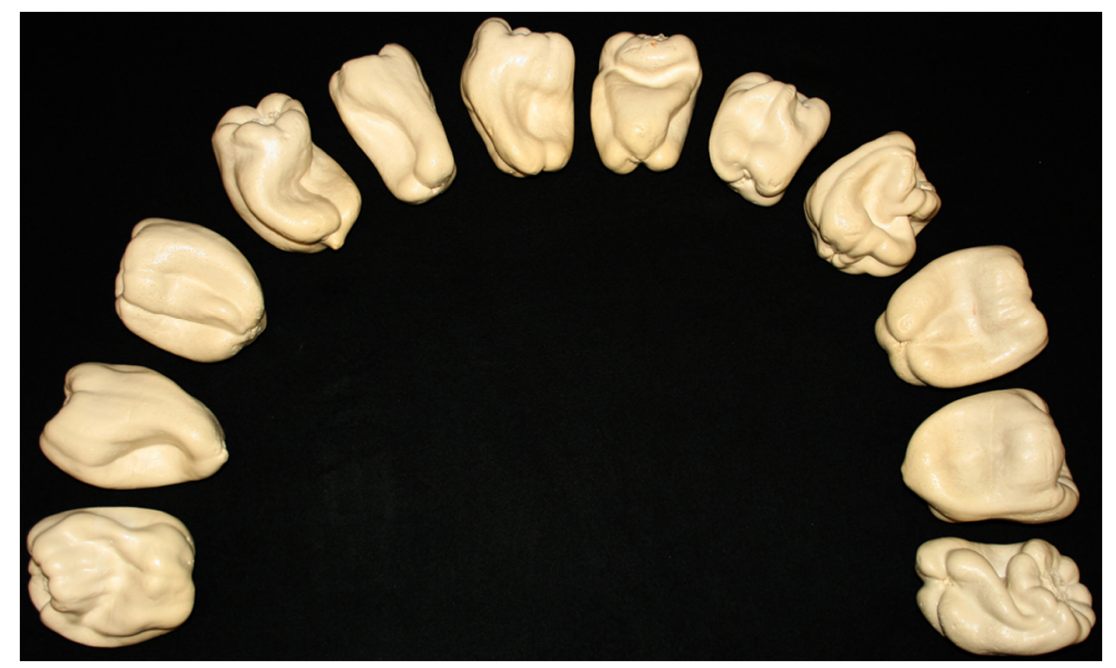

Fig. 3 The 12 naturally shaped objects (replicas of bell peppers, Capsicum annuum) used as stimuli in Experiment 2. Object 13 is located at the left. The objects progress numerically in order from left to right; object 24 , therefore, is located at the far right. 
conditions and on all trials, the computer-rendered objects were randomly oriented in 3-dimensional space and were presented (either statically or in motion) for 15 seconds. The participants' task on each trial was to view a randomly chosen computer-rendered object and then select/ identify a matching physical object (i.e., one that possessed the same 3-D shape) from among the 12 physical bell pepper replicas placed on a table in front of them (the physical bell peppers were located at a $55-\mathrm{cm}$ viewing distance). It is important to note that the participants could see the computer-rendered object and the set of 12 physical bell pepper replicas simultaneously; given that each trial's duration was a lengthy 15 seconds, there was ample opportunity for the participants to perceive the shape of all objects. Each participant made a total of 144 judgments (6 experimental conditions x 12 stimulus objects x 2 repetitions/object/experimental condition).

Participants There were a total of 28 participants. The 14 older participants were the same as those who participated in Experiment 1 . The performance of the older participants was compared to a new sample of 14 younger adults (mean age was 20.5 years, $\mathrm{SD}=2.4$, range $18-25$ years). The participants' visual acuity was good (mean acuity was 0.007 and $0.029 \log$ MAR for the younger and older adults, respectively). All participants gave written consent before participation in the experiment. One younger participant's (age $=21$ years) data were excluded, because she did not conscientiously perform the shape identification task. As a result, her performance was more than 4 standard deviations lower than the average of the other 14 younger adults (the probability that this younger adult's performance came from the same distribution as the other 14 younger adult participants is 0.000017 ). The experiment was approved by the Western Kentucky University Institutional Review Board. Our research was performed in accordance with the Code of Ethics of the World Medical Association (Declaration of Helsinki).

\section{Results and discussion}

Various aspects of the results are shown in Figs. 5, 6, 7 and 8. One can readily see from an inspection of Fig. 5 that the participants' shape recognition performance was much higher in the conditions with motion (i.e., conditions with deforming occlusion boundary contours and/or deforming patterns of specular highlights). One also can see that the amount of improvement associated with object motion, while large for both age groups, differed for the younger and older adults. A threeway analysis of variance (ANOVA) with two within-subjects factors (type of optical information: $\mathrm{BCSH}, \mathrm{BC}$, and $\mathrm{SH}$; presence/absence of motion) and one between-subjects factor (age) was conducted upon the data plotted in Fig. 5. The
ANOVA revealed significant main effects of optical information type $\left(\mathrm{F}(2,52)=13.4, p=0.00002, \eta_{\mathrm{p}}^{2}=0.34\right)$, motion $\left(\mathrm{F}(1,26)=125.8, p<0.000001, \eta_{\mathrm{p}}^{2}=0.83\right)$, and age $(\mathrm{F}(1,26)$ $\left.=15.5, p<0.001, \eta_{\mathrm{p}}^{2}=0.37\right)$, as well as significant interactions of optical information $\mathrm{x}$ motion $(\mathrm{F}(2,52)=3.6, p<0.04$, $\left.\eta_{\mathrm{p}}^{2}=0.12\right)$ and age $\mathrm{x}$ motion $\left(\mathrm{F}(1,26)=13.0, p<0.002, \eta_{\mathrm{p}}^{2}=\right.$ $0.33)$. The optical information $x$ motion interaction occurred, because the effect of motion was larger for the stimulus displays that included specular highlights (conditions $\mathrm{SH}$ and BCSH) and was smaller for the silhouettes that lacked specular highlights (condition $\mathrm{BC}$ ). The age $\mathrm{x}$ motion interaction occurred, because the effect of motion upon the participants' performance was much larger for the younger adults (the younger adults' improvement in performance with motion was 94.7 percent larger than the improvement exhibited by the older adults).

Figure 6 illustrates how the younger and older participants' performance varied across the individual stimulus objects (i.e., bell peppers 13-24). Object 17 was the easiest to recognize for both age groups, whereas objects 13 and 23 were the most difficult to identify. A two-way ANOVA (one withinsubjects factor, object and one between-subjects factor, age; data collapsed across optical information type) demonstrated that the main effect of object was significant $(F(11,286)=$ $\left.31.7, p<0.000001, \eta_{\mathrm{p}}^{2}=0.55\right)$. There was, however, no age $\mathrm{x}$ object interaction $\left(\mathrm{F}(11,286)<1.0, p=0.46, \eta_{\mathrm{p}}^{2}=0.037\right)$, indicating that the effect of object was similar for both younger and older adults.

The younger and older participants' overall confusion matrices are shown in Tables 1 and 2 and are graphically depicted in Fig. 7. The frequencies of the various responses for each of the 12 stimulus objects are indicated. For each of the 66 possible pairs of objects $(13 \& 14,13 \& 15,13 \& 16,13 \& 17, \ldots$, $23 \& 24)$, smaller $(2 \times 2)$ confusion matrices were extracted from Tables 1 and 2 to determine pairwise discriminability/ confusability (Bell \& Lappin, 1973; Norman, Dawson, \& Raines, 2000; Norman, Norman, et al., 2004). The younger and older participants' discriminability for each pair of objects (i.e., d' value) was determined from these individual confusion matrices following the methods described by Macmillan \& Creelman (1991, pp. 7-11). The resulting d' values are plotted in Figure 8. Notice from an inspection of the figure that objects 21 and 24 were frequently confused with each other for both younger and older adults (stimulus was object 21 , but participant chose object 24 as the matching object and vice-versa). Other object pairs (e.g., 17\&21, 17\&24) were highly discriminable for both younger and older adults; yet other object pairs (e.g., 15\&24) produced medium discriminabilities. It is clear from an inspection of Fig. 8 that the younger and older participants perceived the stimulus object shapes very similarly: in fact, the majority of the variance $\left(58.4 \%, r^{2}=0.584\right)$ within the older adults' discriminabilities could be accounted for by the variability of the younger 


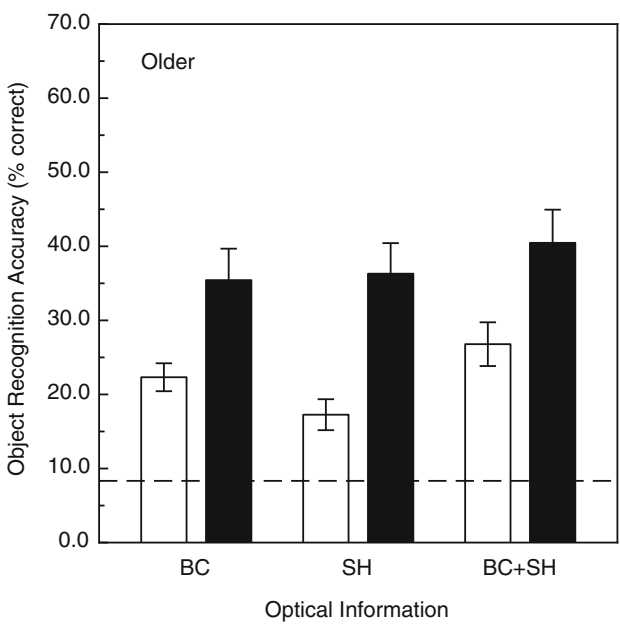

Fig. 5 Results of Experiment 2. The participants' shape recognition accuracies are plotted as functions of stimulus type (objects defined by occlusion boundary contours only, condition $\mathrm{BC}$; objects defined by specular highlights only, condition $\mathrm{SH}$; objects defined by both boundary contours and specular highlights, condition $\mathrm{BCSH}$ ) and the

participants' shape discrimination performance. One also can see that although the younger and older adults exhibited a similar pattern of performance, the older adults were $30 \%$ less sensitive (d' values were $30 \%$ smaller) to variations in the stimulus object shapes.

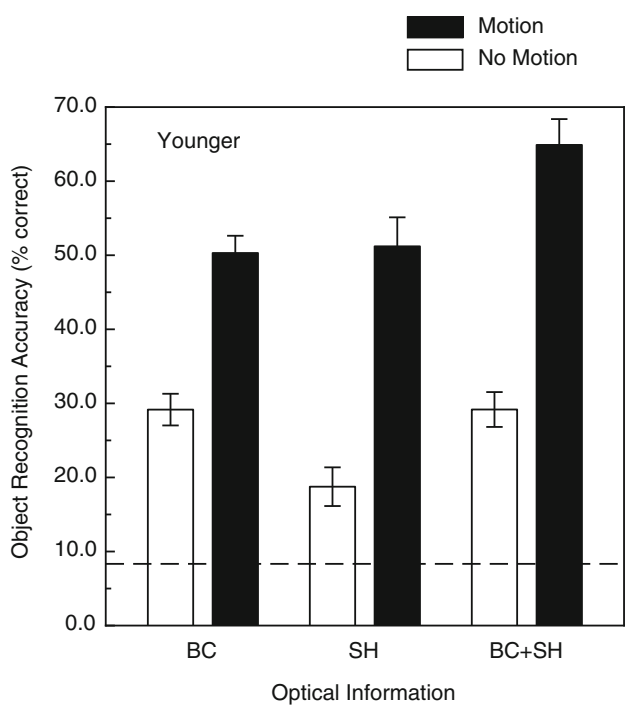

presence/absence of motion (rotation in depth). The dashed lines indicate chance levels of performance. The left and right panels plot the results obtained for the older and younger participants, respectively. The error bars indicate $\pm 1 \mathrm{SE}$.

\section{General discussion}

Previous research (Norman et al., 2006) has shown that older adults can discriminate visual 3-D shape (same versus different judgments) as well as younger adults, at least

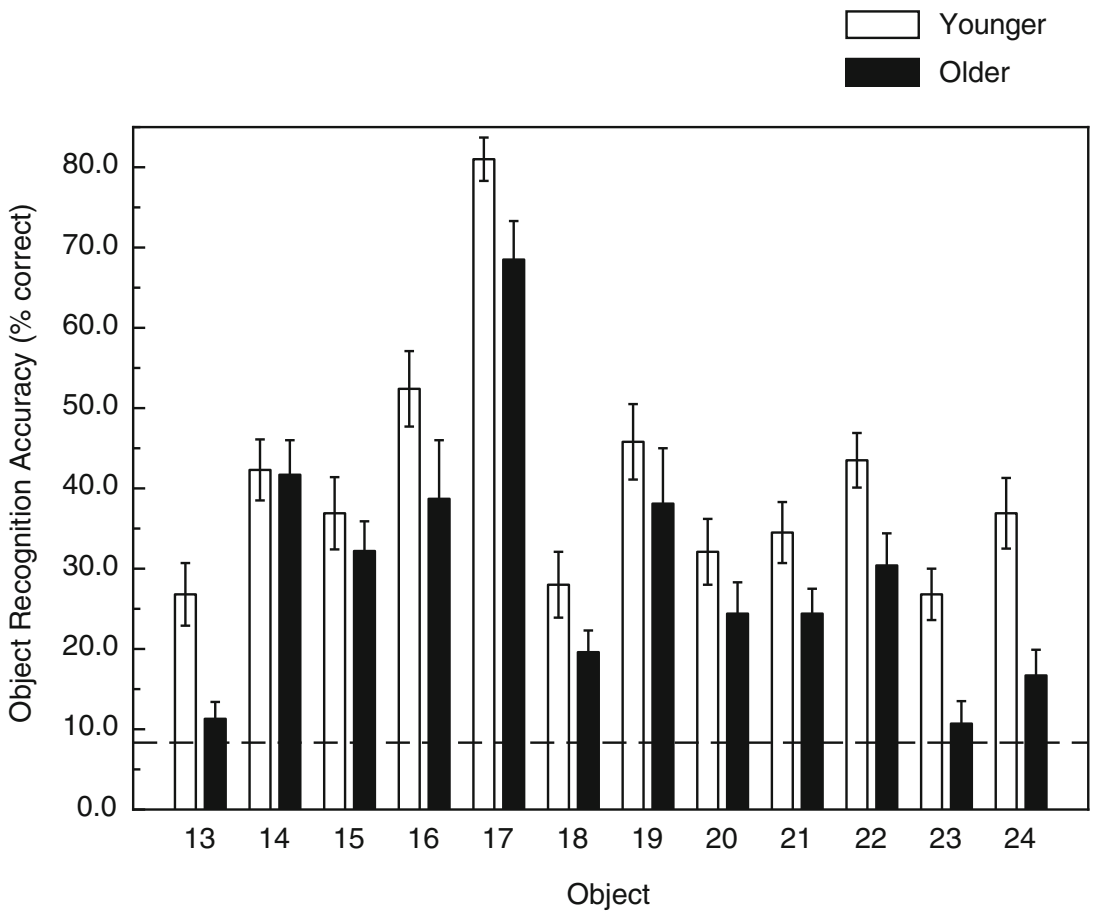

Fig. 6 Results of Experiment 2. The participants' shape recognition accuracies are plotted for each stimulus object (bell peppers 13-24). The dashed line indicates chance levels of performance. The younger and older participants' results are plotted separately (white and black bars, respectively). The error bars indicate $\pm 1 \mathrm{SE}$. 

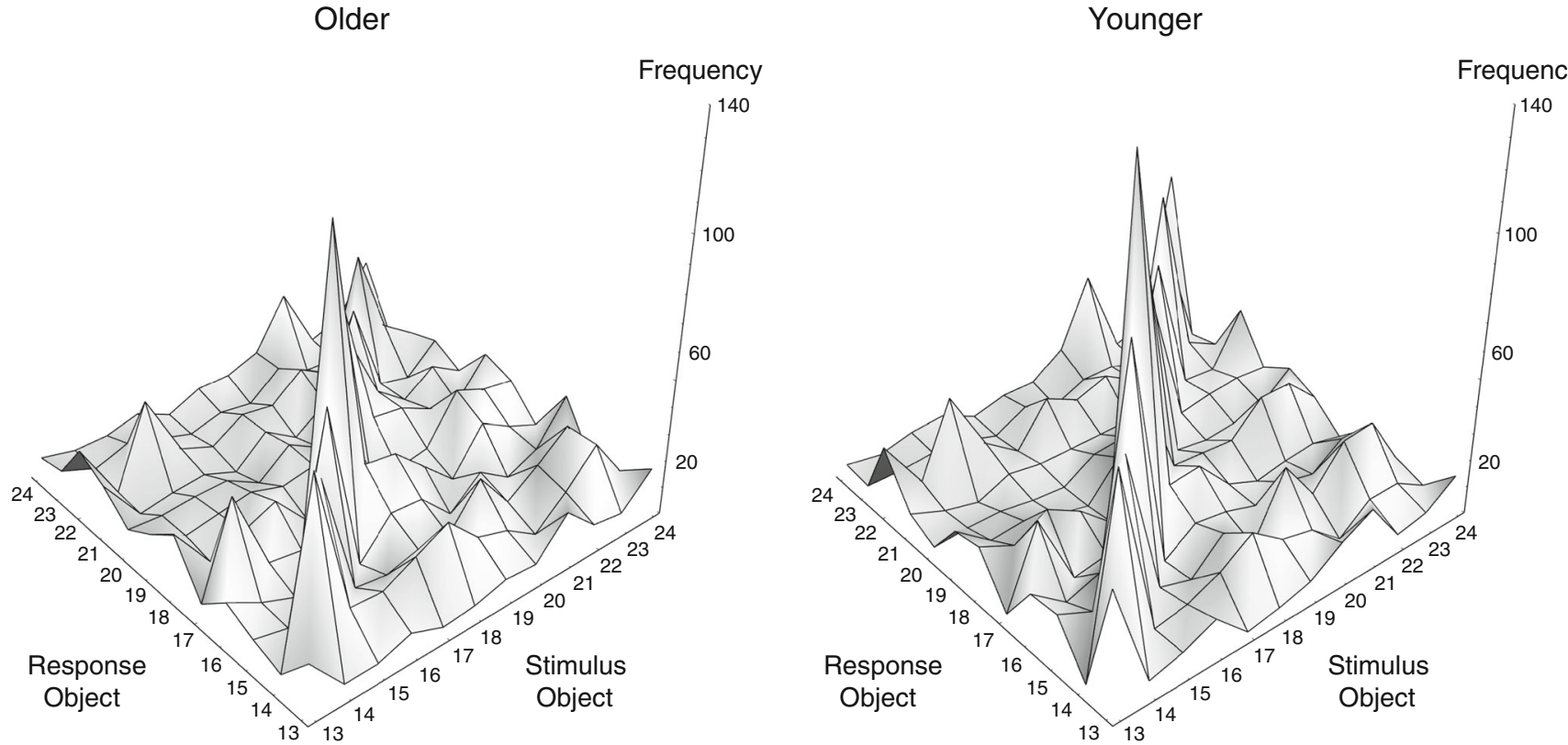

Fig. 7 Results of Experiment 2. Graphical depictions of the confusion matrices presented in Tables 1 and 2; the left and right panels indicate the pattern of object confusions exhibited by the older and younger participants, respectively.

under relatively full-cue conditions (conditions that included motion, occlusion boundary contours, and specular highlights). The results of the current Experiment 2 clearly demonstrate that this previous finding does not extend to shape recognition: Fig. 5 shows that older adults cannot recognize natural object shape as well as younger adults in the conditions employing motion (rotation in depth; $55.5 \%$ and $37.4 \%$ correct overall recognition accuracy for younger and older participants, respectively). Figure 5 does demonstrate that motion facilitates older adults' abilities to recognize object shape (i.e., older adults benefit from the kinetic depth effect), but the magnitude of this facilitation is substantially smaller than that that occurs for younger adults. The findings of Experiment 1 are similar: older adults can recognize 3-D shape when the motions of projected points are the sole

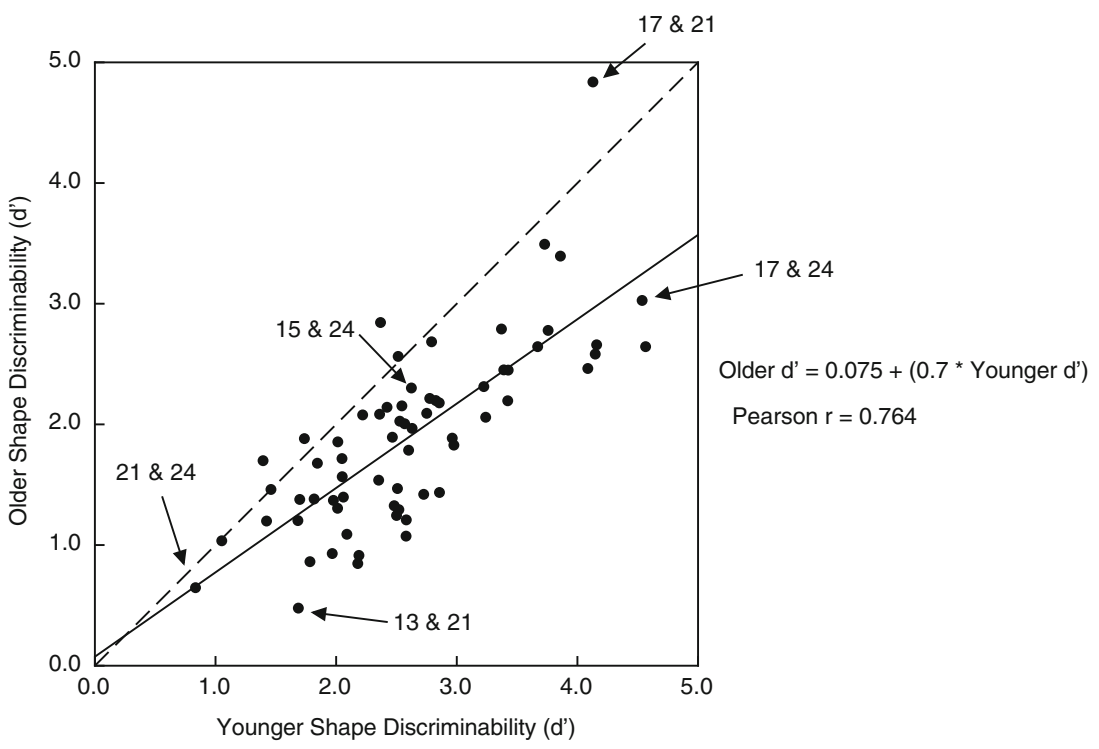

Fig. 8 Results of Experiment 2. The participants' shape discriminabilities (d' values) are plotted for each of the 66 pairs of stimulus objects. The younger and older participant shape discriminabilities are plotted on the abscissa and ordinate, respectively (i.e., $\mathrm{x}$ and $\mathrm{y}$ axes). The solid line indicates the best-fitting linear regression, while the dashed line indicates equivalent performance for younger and older adults. 
Table 1 Confusion matrix, younger adults

\begin{tabular}{|c|c|c|c|c|c|c|c|c|c|c|c|c|}
\hline \multirow[t]{2}{*}{ Response object } & \multicolumn{12}{|c|}{ Stimulus object } \\
\hline & 13 & 14 & 15 & 16 & 17 & 18 & 19 & 20 & 21 & 22 & 23 & 24 \\
\hline 13 & 45 & 5 & 8 & 13 & 1 & 3 & 6 & 14 & 20 & 4 & 6 & 14 \\
\hline 14 & 2 & 71 & 8 & 10 & 15 & 23 & 14 & 11 & 1 & 10 & 10 & 3 \\
\hline 15 & 19 & 13 & 62 & 8 & 4 & 15 & 4 & 27 & 11 & 25 & 26 & 7 \\
\hline 16 & 18 & 8 & 12 & 88 & 3 & 11 & 9 & 5 & 8 & 5 & 13 & 11 \\
\hline 17 & 2 & 30 & 2 & 4 & 136 & 18 & 19 & 6 & 1 & 3 & 4 & 1 \\
\hline 18 & 19 & 13 & 20 & 13 & 2 & 47 & 12 & 11 & 10 & 21 & 21 & 10 \\
\hline 19 & 17 & 7 & 8 & 4 & 1 & 14 & 77 & 5 & 3 & 4 & 6 & 15 \\
\hline 20 & 3 & 4 & 4 & 2 & 1 & 8 & 9 & 54 & 7 & 9 & 10 & 9 \\
\hline 21 & 8 & 3 & 0 & 5 & 3 & 3 & 3 & 3 & 58 & 8 & 7 & 26 \\
\hline 22 & 26 & 7 & 33 & 10 & 1 & 16 & 10 & 14 & 7 & 73 & 12 & 6 \\
\hline 23 & 4 & 4 & 5 & 4 & 0 & 6 & 1 & 6 & 6 & 2 & 45 & 4 \\
\hline 24 & 5 & 3 & 6 & 7 & 1 & 4 & 4 & 12 & 36 & 4 & 8 & 62 \\
\hline
\end{tabular}

source of optical information, but once again there is a substantial adverse effect of age (Fig. 1).

In the current Experiment 1, the 3-D shapes to be recognized were defined by the projected motions of points that had limited temporal lifetimes. Our experiment was similar to one conducted by Andersen and Ni (2008). In their Experiment 2, younger and older participants were required to identify 2-D shapes (circle, square, diamond, and star) that were defined by dynamic occlusion; Andersen and $\mathrm{Ni}$ also manipulated the lifetimes of the points that specified both the moving stimulus surfaces and their background. While Andersen and Ni found that reductions in surface point lifetime resulted in a deterioration in shape identification performance (see their Fig. 6, p. 116), there was no age effect; their younger and older

Table 2 Confusion matrix, older adults

\begin{tabular}{|c|c|c|c|c|c|c|c|c|c|c|c|c|}
\hline \multirow[t]{2}{*}{ Response object } & \multicolumn{12}{|c|}{ Stimulus object } \\
\hline & 13 & 14 & 15 & 16 & 17 & 18 & 19 & 20 & 21 & 22 & 23 & 24 \\
\hline 13 & 19 & 4 & 4 & 8 & 3 & 4 & 7 & 6 & 19 & 8 & 6 & 17 \\
\hline 14 & 6 & 70 & 16 & 15 & 21 & 27 & 15 & 11 & 3 & 13 & 25 & 9 \\
\hline 15 & 10 & 7 & 54 & 8 & 6 & 9 & 8 & 23 & 8 & 16 & 25 & 6 \\
\hline 16 & 15 & 8 & 3 & 65 & 2 & 15 & 14 & 2 & 12 & 4 & 9 & 24 \\
\hline 17 & 6 & 39 & 8 & 6 & 115 & 26 & 24 & 13 & 2 & 18 & 11 & 6 \\
\hline 18 & 24 & 7 & 12 & 17 & 3 & 33 & 12 & 5 & 7 & 24 & 19 & 17 \\
\hline 19 & 16 & 9 & 11 & 13 & 9 & 11 & 64 & 18 & 16 & 10 & 7 & 21 \\
\hline 20 & 10 & 9 & 10 & 10 & 3 & 12 & 5 & 41 & 16 & 7 & 15 & 8 \\
\hline 21 & 19 & 2 & 4 & 5 & 0 & 10 & 2 & 10 & 41 & 7 & 4 & 14 \\
\hline 22 & 25 & 3 & 32 & 8 & 3 & 10 & 8 & 17 & 10 & 51 & 19 & 11 \\
\hline 23 & 10 & 5 & 9 & 5 & 1 & 4 & 2 & 11 & 5 & 4 & 18 & 7 \\
\hline 24 & 8 & 5 & 5 & 8 & 2 & 7 & 7 & 11 & 29 & 6 & 10 & 28 \\
\hline
\end{tabular}

participants performed similarly. The results of the current Experiment 1 (Fig. 1) are clearly different; unlike the analogous experiment of Andersen and $\mathrm{Ni}$, we obtained a large and adverse effect of age regardless of the duration of surface point lifetime. It is thus obvious that aging differentially affects the visual recognition of 2-D and 3-D shapes defined by motion.

In the current Experiment 2, the younger and older participants exhibited very similar patterns of object confusions (compare Tables 1 and 2; also see Figs. 7 and 8). For example, if a participant viewed object 21, they often selected object 24 as possessing the same shape (which was certainly incorrect, because these are entirely different objects that possess mathematically distinct solid shapes); conversely, if a participant viewed object 24, they often believed it was object 21 (because they frequently selected object 21 as the matching object). At this point, it is important to note that the confusions exhibited by our current participants, obtained for visually defined objects, were essentially the same as those obtained in a recent haptic study (Norman, Adkins, Dowell, Hoyng, et al., 2017). Figures 9 and 10 demonstrate these similar patterns of visual and haptic object confusability/discriminability for younger and older participants, respectively. Note that objects 13 and 21 are highly confusable for both vision and haptics. Objects 15 and 24 are moderately confusable for vision and haptics. Finally, note that objects 17 and 21 produce very low confusion and their shapes are highly discriminable for both vision and haptics. This correspondence in how our objects were perceived across the two studies (current Experiment 2 \& Norman, Adkins, Dowell, Hoyng, et al., 2017) indicates that the optical information provided to participants in the current Experiment 2 (specular highlights, occlusion boundaries, and their deformations) is functionally similar to that provided by haptics (sense of active touch). The results shown in Figs. 9 and 10 also are consistent with conclusions made by Snow et al. (2011). These authors used fMRI and found that neuronal activation differed depending upon whether observers viewed "real objects" (i.e., physical solid objects) or photographs of those same objects (which ostensibly present much of the same optical information as the real objects themselves). A careful examination of the current Figs. 9 and 10 reveals that our participants' shape sensitivities (d'values) were frequently higher for haptic stimulus presentation (when participants could feel the actual solid 3-D objects) than for visual stimulus presentation of the same objects when they were computationally rendered as projected images (Fig. 4). Notice, for example, in Fig. 9 that the haptic discriminability obtained for objects 17 and 21 was higher than that obtained for computationally rendered visual images. This superiority for solid stimulus objects over rendered images occurred for most of the 66 pairs of stimulus objects (notice that the slopes of the best-fitting regression lines in both Figs. 9 and 10 were much less than 1.0, indicating that for a given change in shape discriminability for actual solid 


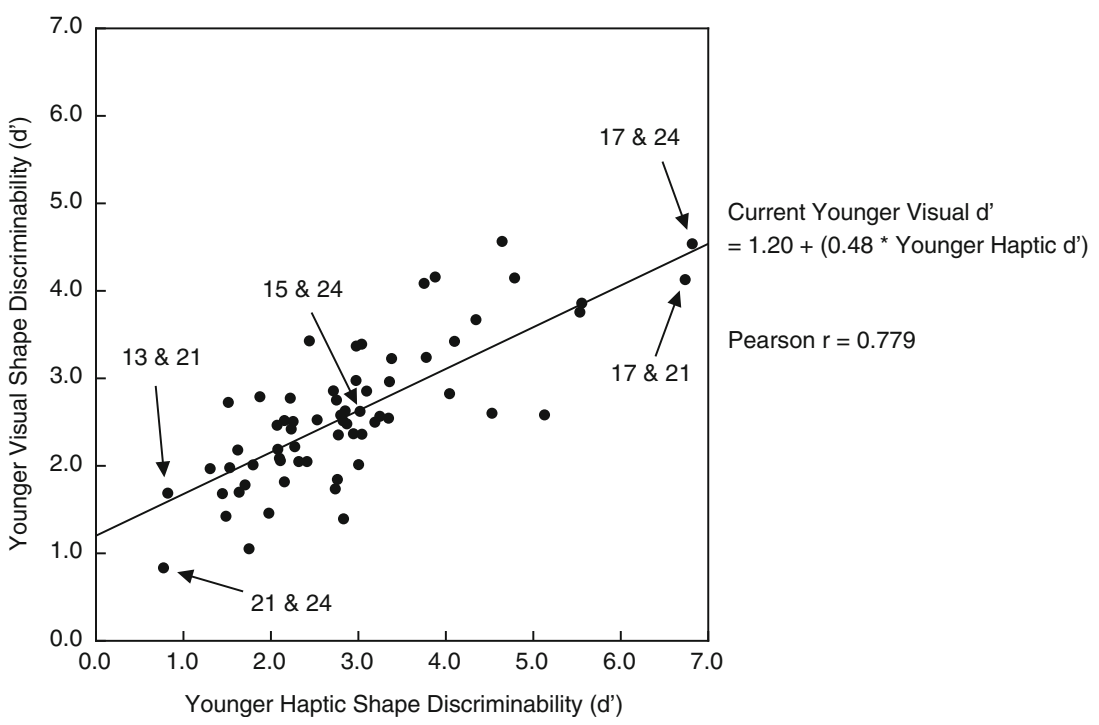

Fig. 9 The younger participants' visual shape discriminabilities for the current Experiment 2 (ordinate, y-axis) are plotted as a function of the haptic shape discriminabilities obtained for younger participants for the same set of objects (13-24) by Norman, Adkins, Dowell, Hoyng, et al. (2017). The solid line indicates the best-fitting linear regression line.

research on the visual perception of distance. Norman, Adkins, Pedersen, Reyes, Wulff, and Tungate (2015) asked younger and older participants to judge environmental distances outdoors. While some individual younger and older participants overestimated the depth intervals and others underestimated the depth intervals, there was no significant effect of age. A similar outcome (i.e., no age effect) occurred for indoor judgments of distance intervals (Norman, Adkins, \& Pedersen, 2016). In another set of studies (Bian \& Andersen, 2013; Norman, Adkins, Norman, Cox, \& Rogers, 2015; Norman, Adkins, Dowell, Shain, Hoyng, \& Kinnard, superior, to younger adults. Consider, for example, past

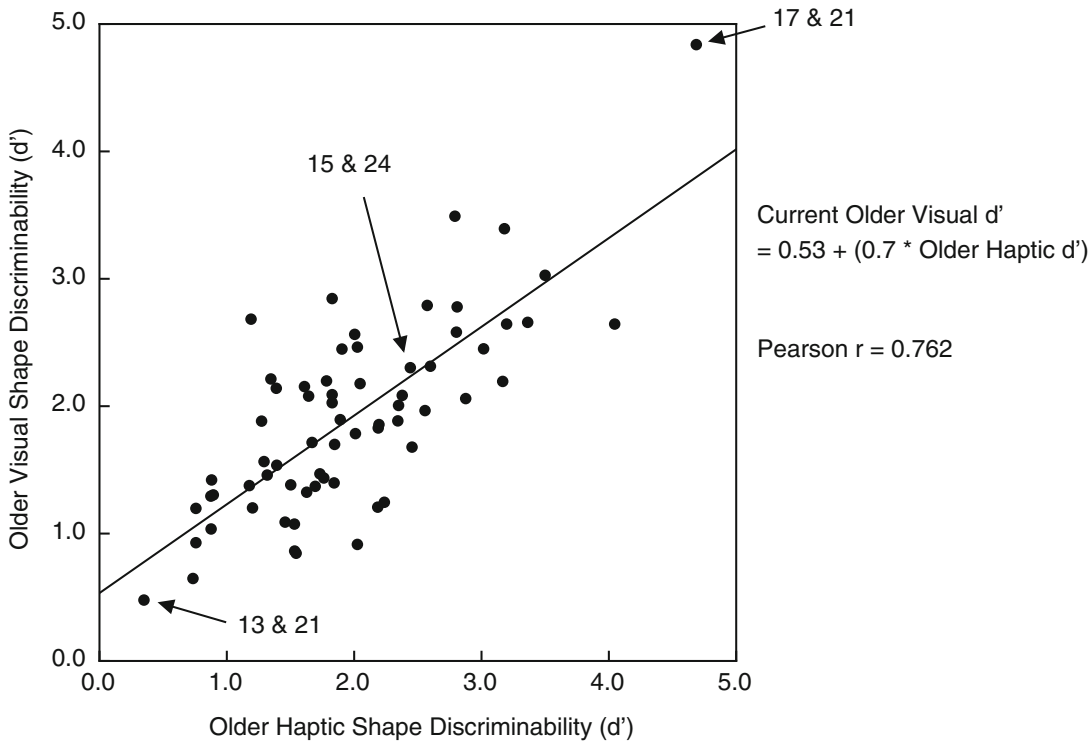

Fig. 10 The older participants' visual shape discriminabilities for the current Experiment 2 (ordinate, y-axis) are plotted as a function of the haptic shape discriminabilities obtained for older participants for the same set of objects (13-24) by Norman, Adkins, Dowell, Hoyng, et al. (2017). The solid line indicates the best-fitting linear regression line. 
2017), older adults were found to exhibit superior performance. In a pioneering study by Bian and Andersen, younger and older adults were asked to estimate egocentric distances in an outdoor grassy field. They found the judgments of older adults to be accurate in absolute terms (no over- or underestimation), and these judgments were superior to those provided by younger adults. The outcome of such studies provide valuable context for the current results. While the current experiments (Figs. 1 and 5) document large and adverse effects of age upon visual shape recognition, it is important to note that performance for other visual tasks is unaffected (or even heightened) by increases in age. The effects of age upon vision, therefore, are task-specific.

\section{Conclusions}

While specular highlights and occlusion boundary contours represent valuable sources of information that enable and support the visual recognition of object shape, the ability to utilize deforming boundary contours and specular highlights deteriorates with increasing age.

\section{References}

Andersen, G. J. (1996). Detection of smooth three-dimensional surfaces from optic flow. Journal of Experimental Psychology: Human Perception and Performance, 22, 945-957. doi:10.1037/00961523.22.4.945

Andersen, G. J., \& Ni, R. (2008). Aging and visual processing: Declines in spatial not temporal integration. Vision Research, 48, 109-118. doi:10.1016/j.visres.2007.10.026

Bell, H. H., \& Lappin, J. S. (1973). Sufficient conditions for the discrimination of motion. Perception \& Psychophysics, 14, 45-50. doi:10. 3758/BF03198616

Bian, Z., \& Andersen, G. J. (2013). Aging and the perception of egocentric distance. Psychology and Aging, 28, 813-825. doi:10.1037/ a0030991

Braunstein, M. L. (1966). Sensitivity of the observer to transformations of the visual field. Journal of Experimental Psychology, 72, 683-689. doi:10.1037/h0023735

Fleming, R. W., Torralba, A., \& Adelson, E. H. (2004). Specular reflections and the perception of shape. Journal of Vision, 4(9), 798-820. doi:10.1167/4.9.10

Hughes, J. F., van Dam, A., McGuire, M., Sklar, D. F., Foley, J. D., Feiner, S. K., \& Akeley, K. (2014). Computer graphics: principles and practice ( $3 r d$ ed.). Upper Saddle River, New Jersey : AddisonWesley.

Kennedy, J. M. (1974). A psychology of picture perception: Images and information. San Francisco, CA: Jossey-Bass.

Kennedy, J. M., \& Silver, J. (1974). The surrogate functions of lines in visual perception: Evidence from antipodal rock and cave artwork sources. Perception, 3, 313-322. doi:10.1068/p030313

Koenderink, J. J. (1984). What does the occluding contour tell us about solid shape? Perception, 13, 321-330. doi:10.1068/p130321

Koenderink, J. J., Kappers, A. M. L., Todd, J. T., Norman, J. F., \& Phillips, F. (1996). Surface range and attitude probing in stereoscopically presented dynamic scenes. Journal of Experimental Psychology: Human Perception and Performance, 22, 869-878. doi:10.1037/0096-1523.22.4.869

Koenderink, J. J., \& van Doorn, A. J. (1976). Visual perception of rigidity of solid shape. Journal of Mathematical Biology, 3, 79-85. doi:10. 1007/BF00307859

Macmillan, N. A., \& Creelman, C. D. (1991). Detection theory: A user's guide. New York: Cambridge University Press.

Norman, J. F., \& Wiesemann, E. Y. (2007). Aging and the perception of local surface orientation from optical patterns of shading and specular highlights. Perception \& Psychophysics, 69, 23-31. doi:10. 3758/BF03194450

Norman, J. F., Adkins, O. C., Dowell, C. J., Hoyng, S. C., Gilliam, A. N., \& Pedersen, L. E. (2017). Aging and haptic-visual solid shape matching. Perception, 46, 976-986. doi:10.1177/0301006617690168

Norman, J. F., Adkins, O. C., Dowell, C. J., Shain, L. M., Hoyng, S. C., \& Kinnard, J. D. (2017). The visual perception of distance ratios outdoors. Attention, Perception, \& Psychophysics, 79, 1195-1203. doi: 10.3758/s13414-017-1294-9

Norman, J. F., Adkins, O. C., Norman, H. F., Cox, A. G., \& Rogers, C. E. (2015). Aging and the visual perception of exocentric distance. Vision Research, 109, 52-58. doi:10.1016/j.visres.2015.02.007

Norman, J. F., Adkins, O. C., Pedersen, L. E., Reyes, C. M., Wulff, R. A., \& Tungate, A. (2015). The visual perception of exocentric distance in outdoor settings. Vision Research, 117, 100-104. doi:10.1016/j. visres.2015.10.003

Norman, J. F., Adkins, O. C., \& Pedersen, L. E. (2016). The visual perception of distance ratios in physical space. Vision Research, 123, 1-7. doi:10.1016/j.visres.2016.03.009

Norman, J. F., Cheeseman, J. R., Pyles, J., Baxter, M. W., Thomason, K. E., \& Calloway, A. B. (2013). The effect of age upon the perception of 3-D shape from motion. Vision Research, 93, 54-61. doi:10.1016/ j.visres.2013.10.012

Norman, J. F., Cheeseman, J. R., Adkins, O. C., Cox, A. G., Rogers, C. E., Dowell, C. J., ... Reyes, C. M. (2015). Aging and solid shape recognition: Vision and haptics. Vision Research, 115, 113-118. doi: 10.1016/j.visres.2015.09.001

Norman, J. F., Crabtree, C. E., Norman, H. F., Moncrief, B. K., Herrmann, M., \& Kapley, N. (2006). Aging and the visual, haptic, and cross-modal perception of natural object shape. Perception, 35, 1383-1395. doi:10.1068/p5504

Norman, J. F., Dawson, T. E., \& Butler, A. K. (2000). The effects of age upon the perception of depth and 3-D shape from differential motion and binocular disparity. Perception, 29, 1335-1359. doi:10.1068/ p3111

Norman, J. F., Dawson, T. E., \& Raines, S. R. (2000). The perception and recognition of natural object shape from deforming and static shadows. Perception, 29, 135-148. doi:10.1068/p2994

Norman, J. F., \& Lappin, J. S. (1992). The detection of surface curvatures defined by optical motion. Perception \& Psychophysics, 51, 386 396. doi:10.3758/BF03211632

Norman, J. F., Norman, H. F., Clayton, A. M., Lianekhammy, J., \& Zielke, G. (2004). The visual and haptic perception of natural object shape. Perception \& Psychophysics, 66, 342-351. doi:10.3758/ BF03194883

Norman, J. F., Phillips, F., Cheeseman, J. R., Thomason, K. E., Ronning, C., Behari, K., Kleinman, K., Calloway, A. B., \& Lamirande, D. (2016). Perceiving object shape from specular highlight deformation, boundary contour deformation, and active haptic manipulation. PLOS ONE, 11(2): e0149058. doi: 10.1371/journal.pone.0149058

Norman, J. F., \& Raines, S. R. (2002). The perception and discrimination of local 3-D surface structure from deforming and disparate boundary contours. Perception \& Psychophysics, 64, 1145-1159. doi:10. 3758/BF03194763

Norman, J. F., Todd, J. T., \& Orban, G. A. (2004). Perception of threedimensional shape from specular highlights, deformations of 
shading, and other types of visual information. Psychological Science, 15, 565-570. doi:10.1111/j.0956-7976.2004.00720.x

Norman, J. F., Todd, J. T., \& Phillips, F. (1995). The perception of surface orientation from multiple sources of optical information. Perception \& Psychophysics, 57, 629-636. doi:10.3758/BF03213268

Shreiner, D. (2010). OpenGL Programming Guide (7th ed.). Upper Saddle River, NJ : Addison-Wesley.

Snow, J. C., Pettypiece, C. E., McAdam, T. D., McLean, A. D., Stroman, P. W., Goodale, M. A., \& Culham, J. C. (2011). Bringing the real world into the fMRI scanner: Repetition effects for pictures versus real objects. Scientific Reports, 1, 130. doi:10.1038/srep00130
Todd, J. T. (1985). Perception of structure from motion: Is projective correspondence of moving elements a necessary condition? Journal of Experimental Psychology: Human Perception and Performance, 11, 689-710. doi:10.1037/0096-1523.11.6.689

Todd, J. T., \& Norman, J. F. (1991). The visual perception of smoothly curved surfaces from minimal apparent motion sequences. Perception \& Psychophysics, 50, 509-523. doi:10.3758/BF03207535

van Damme, W. J. M., \& van de Grind, W. A. (1996). Non-visual information in structure-from-motion. Vision Research, 36, 3119-3127. doi:10.1016/0042-6989(96)00060-0 